\title{
PRELIMINARY REPORT ON THE MBE-4, AN EXPERIMENTAL MULTIPLE- BEAM INDUCTION LINEAR ACCELERATOR FOR HEAVY IONS*
}

\author{
A. I. Warwick, D. E. Gough, and H. Meuth \\ Lawrence Berkeley Laboratory \\ 1 Cyclotron Road \\ Berkeley, Califomia 94720
}

November 1988

\section{DSCEAIMER}

\begin{abstract}
This report we preparad as an acoownt of wort spoesored by an abeacy of the United States

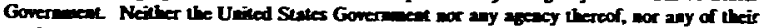

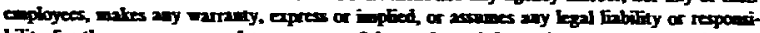

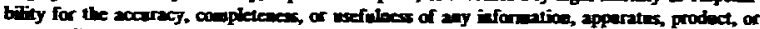

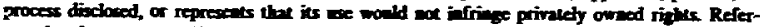
eace hereil to any specific conercial product, process, or service by trade mane, trademart,

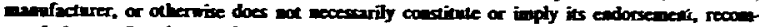

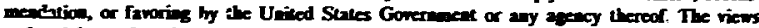

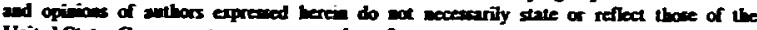
Unied Stres Gorernacit or any aceacy thereof.
\end{abstract}

* This work was supported by the Director, Office of Energy Research, Office of Basic Energy Sciences, Advanced Energy Projects Division, U.S. Department of Energy under Contract No. DE-AC03-76SF00098. 
LBL-28529

HIFAR Note-222

\title{
PRELIMINARY REPORT ON MBE-4, AN EXPERIMENTAL MULTIPLE-BEAM INDUCTION LINEAR ACCELERATOR FOR HEAVY IONS
}

\author{
A.I. WARWICK, DE. GOUGH and H. MEUTH \\ Lawrence Berkeley Laboratory, Berkeley, CA 94720, U.S.A.
}

\begin{abstract}
A small-scale experimental accelerator called MBE-4 has been constructed to demonstrate the principle of a current-amplifying induction linac for multiple beams of heavy ions. Four beams of $\mathrm{Cs}^{1+}$, initially at $200 \mathrm{keV}$ and each with a current of $10 \mathrm{~mA}$ have been accelerated and amplified to a kinetic energy of $700 \mathrm{keV}$ and currents of $90 \mathrm{~mA}$ apiece. Transverse focusing is achieved by means of electrostatic quadrupoles; longitudinally the current is amplified and the beam bunch is held together against the space charge forces by special time dependent accelerating fields. We report on the meihods developed for designing and implementing the accelerating pulses and on measurements of the transverse and longitudinal emittance of the accelerated beams. Current fluctuations and the longitudinal emittance are initially almost zero and increase as acceleration errors are accumulated. We discuss the final longitudinal emittance and the current fluctuations in the experiment in terms of their acceptability for a large heavy-ion-fusion driver.
\end{abstract}

\section{INTRODUCTION}

If heavy ion beams $(A=200)$ are to be used to drive inertial fusion, a large current is required (several $\mathrm{kA}$ ) at a linetic energy per nucleon or about $50 \mathrm{MeV} / \mathrm{u}$.

An induction linac conld efficiently accelerate high current beams of heavy ions. Because the initial velocity wonld be non-relativistic $\left(\beta=10^{-2}\right)$, the head and tail of an initially long bunch $(60 \mathrm{~m})$ could be differentially accelerated by time-dependent accelerating pulses so that the current would be amplified during acctleration.

The high beam current would strain the technological limits of transverse focusing if transported in a single channel. Multiple b.ams are preferred instead individnally focused by electrostatic lenses at first, then by magnetic lenses as the velocity increases.

Longitudinal control must be provided by specially time-dependent accelerating fields which would accelerate the extreme ends of the bunch in such a way as to 
counteract the longitudinal space charge forces. These forces would otherwise elongate the bunch.

This driver scenario bas been studied extensively and reported elsewhere. ${ }^{1}$ In this paper we report the performance of a small scale experiment to demonstrate the principies of current amplification and acceleration in a multiple beam ion induction linac.

MBE-4 has four beams. This is the smallest reasonable number with which to demonstrate a multiple beam linac. Singly-charged cesium ions are used because of the availability of high quality ion sources. The initial kinetic energy is $200 \mathrm{keV}$ and each beam bunch is injected with a current of $10 \mathrm{~mA}$ and a $2.5 \mu$ s duration. The initial line charge-density in each beam is thus $1.9-10-8 \mathrm{~cm}^{-1}$, about 14 times smaller than at the beginning of a representative driver 1 .

MBE-4 uses electrostatic quadrupoles for focusing throughout its length, as may be used in the beginning of a driver. 1

In order to avoid bunch lengthening, acceleration of the head of the bunch does not begin until the bunch is ertirely inside the linac. MBE-4 is 14 times longer than the initial beam bunch, the length of which is short $(1 \mathrm{~m})$ compared to that in a driver $(60 \mathrm{~m})$. The duration of the bunch (the time it takes to pass a given point) determines the amouni of ferromagnetic material required for the induction cores. Our short bunch allows the experiment to be built at reasonable cost. The short bunch in MBE-4 means that a significant portion of a driver, several hundred meters of its length, can be modelled using a vigorous schedule of acceleration and current amplification in the 14-m length of the experiment.

After about $400 \mathrm{~m}$ in a driver ${ }^{1}$ the kinetic energy would have been increased by about a factor of 10 and the current by about a factor of 5 . In the length of MBE-4, using a vigorous schedule, the kinetic energy is increased by a factor of 3.5 and the current by a factor of 9 . Thus for a given increase of kinetic energy the current amplification is greater in MBE-4 than in a driver. Furthermore, each pulser provides a much larger relative increase of the kinetic energy than in a driver, thus the subdivision of the acceleration is much coarser. MBE-4 is then a stringent test of longitudinal control through application of shaped accelerating voltages. 
Table 1

Comparison of the parameters of MLe:- with the a recent driver design

\begin{tabular}{|c|c|c|c|c|c|c|}
\hline & MBE-4 & MBE-4 & $\frac{\text { MBE-4 }}{\text { (gentle) }}$ & $\frac{\text { DRIVER }}{\text { (vigorous) }}$ & DRIVER & DRIVER \\
\hline $\begin{array}{l}\text { longitudina' } \\
\text { position (z) }\end{array}$ & $?=0 \mathrm{~m}$ & $z=14 m$ & $z=14 \mathrm{~m}$ & $\mathrm{z}=0 \mathrm{~m}$ & $z=400 \mathrm{~m}$ & $z=4 \mathrm{~km}$ \\
\hline charge number & 1 & 1 & 1 & 3 & 3 & 3 \\
\hline mass numi.t. & 133 & 133 & 133 & 200 & 200 & 200 \\
\hline lineticeicx6y & $0.2 \mathrm{MeV}$ & $0.62 \mathrm{MeV}$ & 0.70MeV & IOMeV & I0OMeV & $10 G e V$ \\
\hline $\begin{array}{l}\beta \\
\text { number of beams }\end{array}$ & $\begin{array}{l}1.8 .10^{-3} \\
4\end{array}$ & $\begin{array}{l}3210^{-3} \\
4\end{array}$ & $\begin{array}{l}3.4 .10^{-3} \\
4\end{array}$ & $\begin{array}{l}10^{-2} \\
64\end{array}$ & $\begin{array}{l}32.10^{-2} \\
16\end{array}$ & $\begin{array}{l}3210^{-1} \\
16\end{array}$ \\
\hline $\begin{array}{l}\text { current per beam } \\
\text { line charg: CmI } \\
\text { overall bunch length } \\
\text { overnll bunch duration } \\
\text { wrve speed"ms }\end{array}$ & $\begin{array}{l}10 \mathrm{~mA} \\
1.9-10^{-8} \\
1.3 \mathrm{~m} \\
2.5 \mathrm{\mu s} \\
1.7 \cdot 10^{4}\end{array}$ & $\begin{array}{l}\$ 35 \mathrm{~mA} \\
\$ 3.6-10^{8} \\
0.86 \mathrm{~m} \\
900 \mathrm{~ns} \\
+23-10^{4}\end{array}$ & $\begin{array}{l}\text { tg } \mathrm{mA} \\
18.6-10^{-8} \\
0.42 \mathrm{~m} \\
400 \mathrm{~ns} \\
\$ 3.6-10^{4}\end{array}$ & $\begin{array}{l}0.78 \mathrm{~A} \\
2.6-10^{-7} \\
60 \mathrm{~m} \\
20 \mathrm{\mu s} \\
8.8-10^{4}\end{array}$ & $\begin{array}{l}\zeta 15.6 \mathrm{~A} \\
1.6-10^{-6} \\
38.4 \mathrm{~m} \\
41 \mathrm{~s} \\
2.2-10^{5}\end{array}$ & $\begin{array}{l}625 \mathrm{~A} \\
6.5-10^{-6} \\
9.6 \mathrm{~m} \\
100 \mathrm{~ns} \\
4.4-10^{5}\end{array}$ \\
\hline
\end{tabular}

-The speed ef a bongitudinal space-charge wave in a irame moving with the bunch, usitg an empirically determined value of $4 \pi \mathrm{re} g=22$ (see section 4.3).

tPeak vilue.

לBeams merge transversely in sets of four, so that the current per beam increases by a factor of four at this poin.

The essence of MBE-4 is the generation and application of the shaped accelerating pulses and the meastrement of the longitudinal beam dynamics that results. Imperfect pulies give rise to kinetic energy errors in the bunch which propagate as space charge waves and can be seen downstream as fluctuations in the beam current as the bunch passes a measuring station. At the end of the accelerator the kinetic energy profile of the bunch is measured to determine the degradation of the longitudinal emittance.

Although MBE-4 can be scaled for comparison to a driver in some respects, it is not a high-current accelerator. One particularly important point which is not addressed is the interaction of fluctuating hign current beams with the structure impedance.

\section{GENERAL DESCRIPTION OF THE ACCEIERATOR}

Figure 1 is a schematic diagram of the linac. The four-beam diode and its four thermionic emitters have been described elsewhere.2,3 The diode is mounted on a $200 \mathrm{kV}$ Marx generator whis:t pulses for $3 \mu \mathrm{s}$. After emerging from the diode the beams can be individually steered ${ }^{3}$ to correct for mechanical misalignments of the diode, this occurs at the location marked M0 on Figure 1. After the steerers, the four beams enter the first four-fold electrostatic quadrupole lens. ${ }^{4}$ The strengths of the first 8 fourfold lenses are individually adjustable to allow the beams to be matched 5 from the diode exit, where they are round and divergent, into the alternating gradient lattice.

The diagnostic station labelled gap 0 on Figure 1 represents the beginning of the linac. Here the initial properties of the four bearn bunches are measured. There follow 


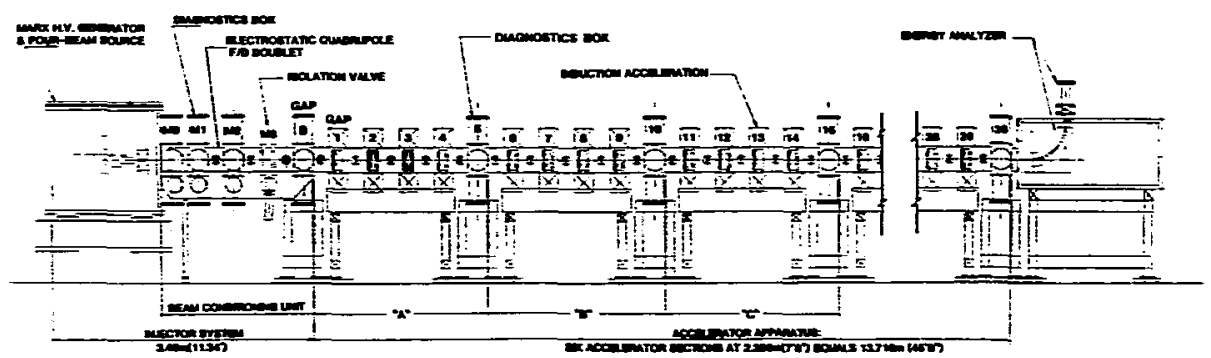

Figure 1. A schematic layout of the accelerator.

six sections of linac, each consisting of five lattice periods. The gaps between quadrupole dorblets are labelled 1 to 30 . Gaps 1-4, 6-9, 11-14, 16-19, 21-24 and 2629 are accelerating stations, every fifth gap is a diagnostic station. At each diagnostic location the transverse emittance and the current can be measured as functions of time as the bunch passes. At the beginning (gap 0) and at the end (gap 30) of the linac an electrostatic energy analyzer measures the kinetic energy as a function of time.

Each accelerating station has a 'core-assembly' which is a set of silicon-steel and nickel-iron toroids surrounding a vacuum insulator through which the four beams pass. The toroids are looped by the primary arive circuit into which a capacitor is discharged as the bunch passes. The design of these pulsers ${ }^{6}$ will be described below. From the point of view of beam dynamics, a time dependent voltage appears across the vacuum insulator so that ions in the bunch are accelerated differently depending upon the time when they arrive at the acceleration gap.

\section{BEAMS WITHOUT ACCELERATION}

In order to assess the degradation of beam quality during acceleration and current amplification, we must first see how the beams behave when simply drifting through the linac with the accelerating fiekds tumed off. At the same time we can determine the initial conditions for the problem of designing the accelerating fulses by measurements at gap 0.

\subsection{Initial current}

Figure 2 shows the current in one of the four beams as a function of time as the bunch passes gap 0 . The head of the bunch is on the left and the time scale is referred to an arbitrary origin which will be common to all the data shown in this paper. The current in the bunch shows an early enhancement, then settles to a uniform value of approximately $10 \mathrm{~mA}$ throughout the rest of the bunch. The enhancement is due to the mismatch between the diode transit time (500 ns) and the Marx pulse risetime. The Marx 


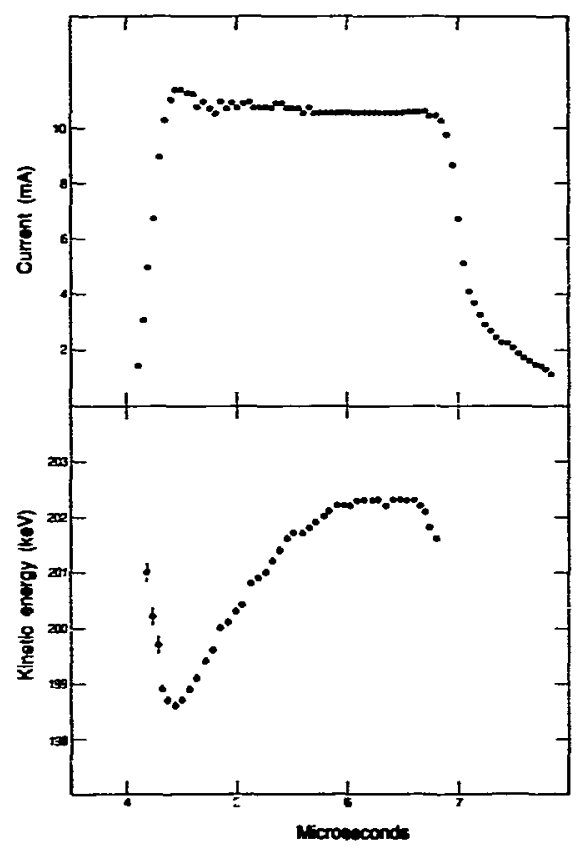

Figure 2. The initial conditions for the longitudinal dynamics. The upper part of the figure shows the current in one beam, the lower part shows the kinetic energy. Both quantities are measured at gap $\mathbf{0}$ and plotted as a function of the time of arrival at this diagnostic station.

pulse risetime has been optimized, subject to practical constraints, to minimize this transient effect. Figure 3 shows oscilloscope traces of the current of the four beams at gap 0 . All beams look the same, with the magnitude of the current varying by $\pm 8 \%$ from beam to beam. These oscilloscope traces show many bunches superimposed. There is essentially no shot-to-shot variation. This is important not only from the point of view of diagnostics, but also from the point of view of beam quaity, since variations between one shot and the next are as damaging as variations within a single bunch.

\subsection{Initial kinetic energy}

The kinetic energy of the bunch as a function of time can be measured with an electrostatic analyzer located at gap 0 . Figure 2 shows the results. The expanded energy scale shows the combined effects of the diode transient and the Marx risetime. This small energy variation from head to tail is measured in several shots, but again the shot-to-shot variation is negligible. 

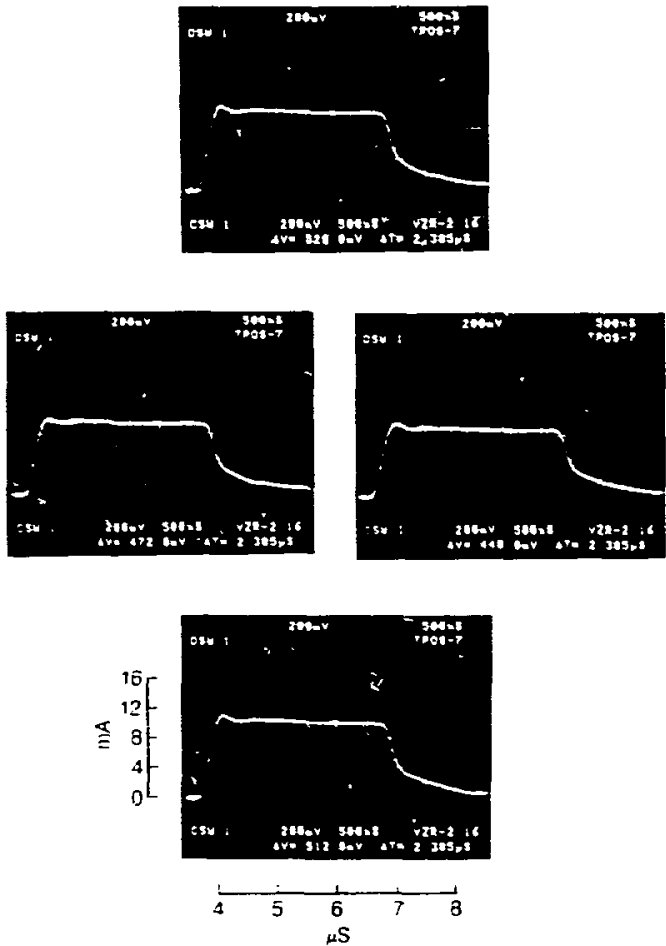

Figure 3. Oscilloscope traces of the current waveforms as the beams pass gap 0 .

\subsection{Transmission}

The four beams. each with a current of $10 \mathrm{~mA}$ and $200 \mathrm{keV}$ kinetic energy, are transponed througi: the MBE-4 latice $a$ ith yero-curem betauron phase-advance of 60 degrees per quadrupole period. Table 2 fiow the fattice parameters.

\section{Table 2}

\section{Quadnoole lanice narameters (10 mA $200 \mathrm{keV}$ )}

aperture radius

quadrupole voltage effective occupancy

period length
27 nis...

$16.1=2$ : wo poles at ground potential) 57

I 2 , ? 
In a K-V beam with $\pi \mathrm{eN}_{\mathrm{N}}=1 \cdot 5 \cdot 10^{-7} \pi \mathrm{m}$ rad (as measured at gap 0 , including halo, see section 3.6) and a current of $10 \mathrm{~mA}$, the betatron tune is depressed from 60 degrees to 12 degrees.

Beam currents are measured by Faraday cups in which the secondary electron effects are suppressed by electric fields. By digitizing the signal and integrating numerically, the total charge in the bunch can be measured at each diagnostic location. Figure 4 shows the measured transmission from gap 0 to gap 30. There are some transverse losses because the aperture is full during matching upstream of gap $0(4 \%)$.

The beam has a halo in transverse phase-space at gap 0 . These halo particles represent a few percent of the intensizy and are presumably lost early in the accelerator because the halo is not visible further down the linac and the emittance is reduced (see section 3.6). These losses are not seen clearly in Figure 4 because these integrated charge measurements also show the loss of the low energy tail, which is generated in the diode as a transient turn-off effect. The low energy tail particles may not actually be lost but are left behind by the drifting bunch until they are undetectable above the electronic noise.

Taking :hese two effects into account there are no other losses observed.

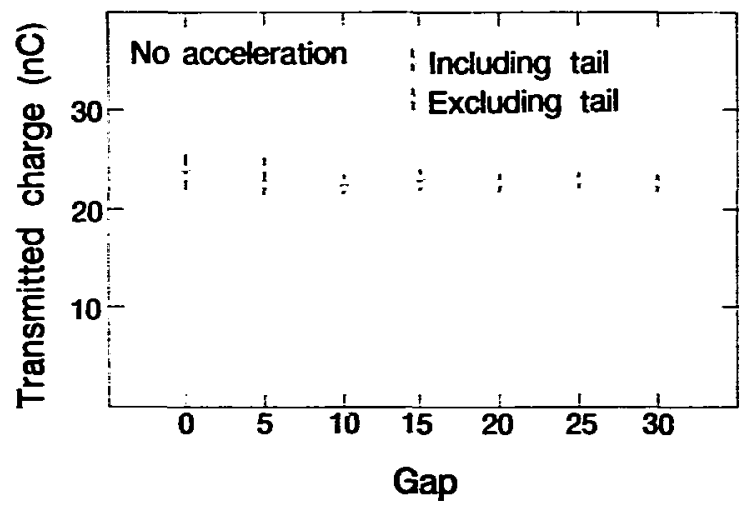

Figure 4. Transmission in MBE-4 without acceleration. The transmitted charge is measured and plotied at each diagnostic station.

\subsection{Final current}

Figure 5 shows the current vaveform for one beam after drifting to gap 30 . Longitudinal space charge forces have distended the ends of the buncin ${ }^{7}$ leaving only the central portion with the initial current of $10 \mathrm{~mA}$. 


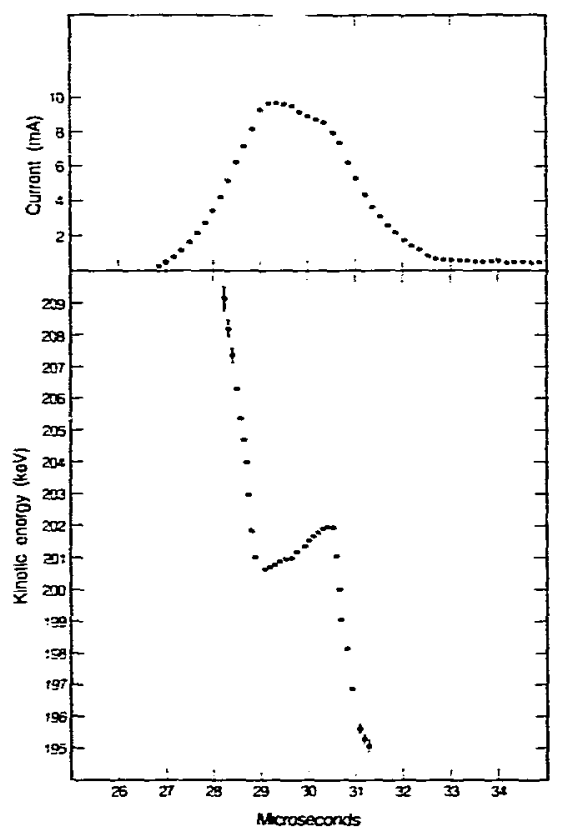

Figure 5. Current and kinetic energy waveforms at gap $\mathbf{3 0}$ after drifting through the linac. The upper part of the figure shows the current in one beam, the lower part shows the kinetic energy.

\subsection{Final kinetic energy}

Figure 5 shows the kinetic energy waveform of the bunch after drifting to gap 30. Here too ore can see the effects of the longitudinal space-charge forces accelerating the head of the bunch and decelerating the tail.

\subsection{Transverse emittance}

The un-normalized transverse r.m.s. emittance is obtained from the measured projection of the phase-space density on the $x-x^{\prime}$ plane, where $x$ is the transverse coordinate and $x^{\prime}$ is its derivative with respect to $z$, the beam direction. Thus,

$$
\varepsilon_{\mathrm{rms} . \mathrm{s}}=\left[\left\langle\mathrm{x}^{2}\right\rangle\left\langle\mathrm{x}^{2}\right\rangle-\left\langle x x^{\prime}\right\rangle^{2}\right]^{1 / 2} .
$$

The normalized emittance has the dependence on the beam velocity renoved:

$$
\varepsilon_{\mathbf{N}}=4 \beta \gamma \varepsilon_{\mathrm{r} . \mathrm{m} s} .
$$


The transverse emittance is measured carefully at the diagnostic gaps; $0,5,10$, $15,20,25$ and 30. Our technique 8 involves slits and detectors which move through the beam profile and about 1000 shots are required for a complete measurement of one beam in one transverse phase space plane, at one location.

Figure 6 shows emittance plots in one plane for the longitudinal center of the bunch at gap 0 and gap 30 . The superimposed ellipses represent the phase-space distributions of $\mathrm{K}-\mathrm{V}$ beams ${ }^{9}$ with the same r.m.s. radii, divergence and emittance. The emittance is larger at gap 0 ( $\varepsilon_{\mathrm{rm} . \mathrm{s}}=20 \mathrm{~mm}$ rarad, including about $95 \%$ of the intensity) because of the presence of a halo, which represents a few percent of the intensity. These halo particles are lost early in the linac. Subsequently the emittance maintains the same lower value $\left(\varepsilon_{\mathrm{r} . \mathrm{m} . \mathrm{S}}=12 \mathrm{~mm}\right.$ mrad, including about $95 \%$ of the intensity) to the end of the linac.

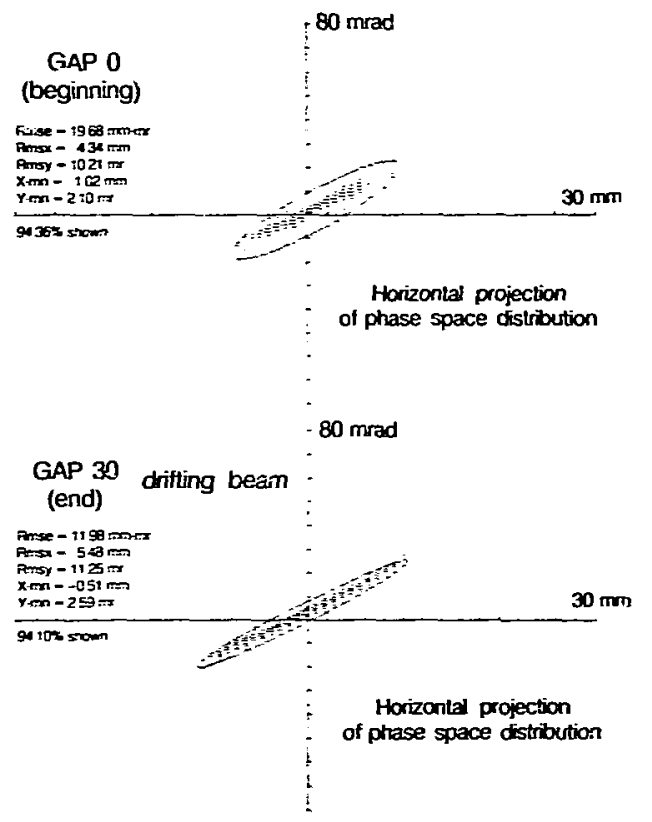

Figure 6. Measurements of the horizontal transverse phase space projection of a drifting beam. Measurements are shown at the beginning (gap 0 ) and at the end (gap 30) of the linac. The ellipses on the emittance plots show the phase space distribution of a $\mathrm{K}-\mathrm{V}$ beam with the same first and second moments as the measured distribution. Approximately $95 \%$ of the intensity is included in these emittance plois. The larger values of emittance at gap 0 are due to low intensity beam halo which is not transmitted through the linac. 
Figure 7 shows the transverse profiles of the beam bunch as a function of time of arrival at detectors located in gaps 0 and 30 . These profiles are obtainet by scanning a slit across the beam and would be elliptical for a uniform beam.

At gap 0 the bunch is cylindrical but non-uniform and somewhat hollow. This follows exposure to non-linear transverse fields in the diode and matching section, where the beam fills the aperture. The transverse profiles change continuously through the linac.

At gap 30 the bunch ends have been distended by the longitudinal space-charge force and the radius decreases as the current decreases towards the ends of the bunch. The beam is hollow midway through the length of the bunch but fairly uniform in the distended bunch ends.

\section{Horizontal Beam Profile}
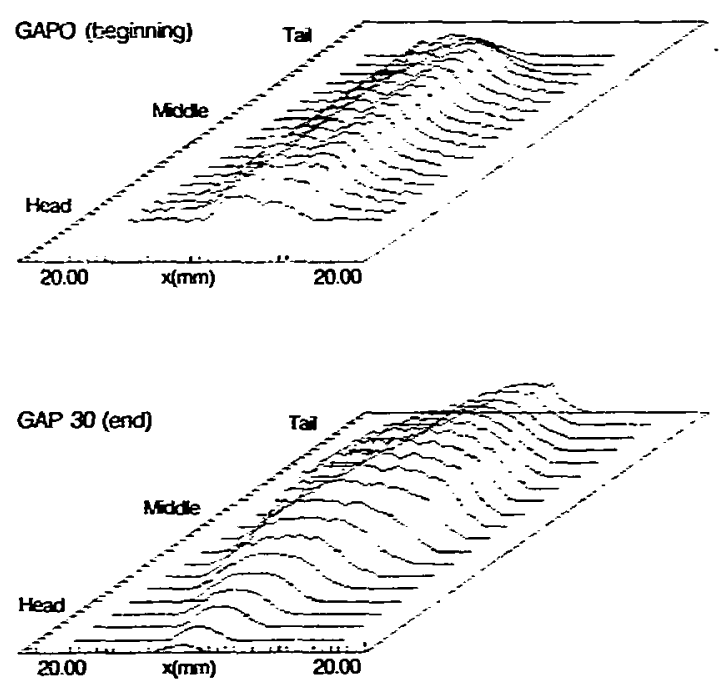

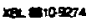

Figure 7. Transverse beam profiles through the length of the bunch at gap 0 , and drifting to gap 30 . These profiles are measured by scanning a slit across the beam and would be elliptical if the beam were uniform. 
Figure 8 shows the r.m.s. properties of one of the beams at gap 0 and at gap 30 , as a function of time as the bunch passes.
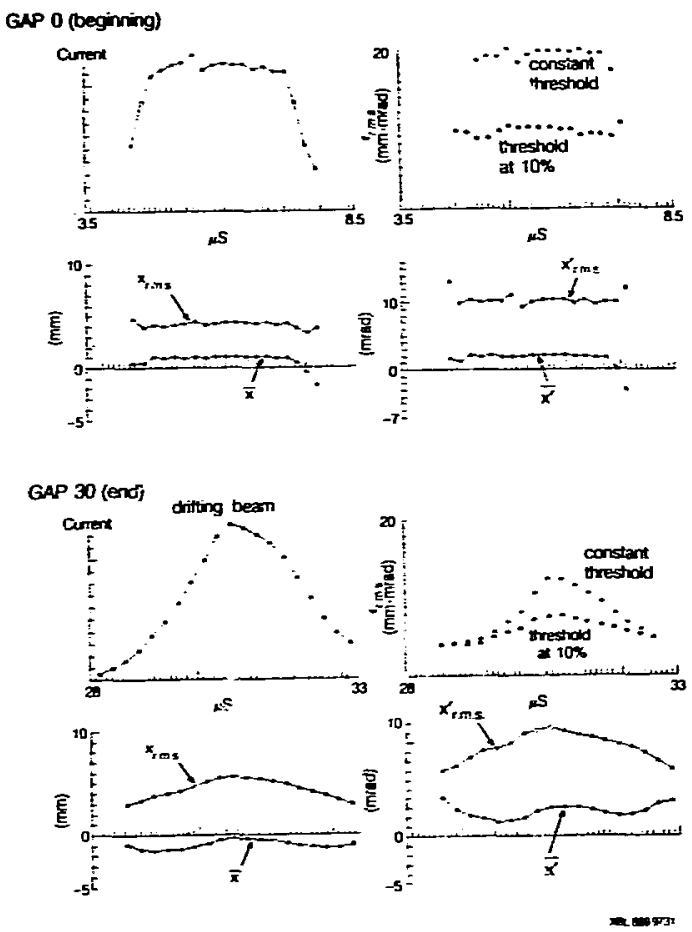

Figure 8. Parameters of the horizontal phase space projection of a drifing beam as a function of position in the bunch. The top part of the figure shows measurements at the beginning of the linac (gap 0), the lower part at the end (gap 30).

Before extracting these r.m.s. quantities it is essential to establish a phare-space density threshold below which signals from the detectors are ignored. Otherwise electronic noise appears as a false, low density population of the transverse phase space, uniform across the scanned phase-space area.

One can see again the larger value of the emittance at gap 0 due to halo which is not transported through the linac. 
The r.m.s. quantities, particularly the r.m.s. emittance, are sensitive to the magnitude of this threshold, which inevitably excludes a small fraction of the beam. The emittance is plotted in Figure 8 with a constant threshold, at the lowest level necessary to eliminate noise. This constant threshold level is fixed throughout the duration of the bunch and at gap 30 results in a value of $\varepsilon_{\mathrm{r} . \mathrm{m} . s}$. biased towards lower values in the distended bunch ends, where the beam current decreases and the constant threshold removes an increasing fraction of the real particles.

The emitrance is also plotted for a threshold at a fixed fraction (10\%) of the maximum phase space density as it changes through the length of the bunch. This

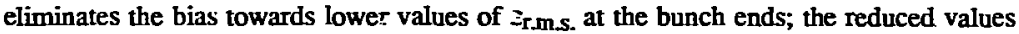
persist.

Our expectation was that the emittance would be unchanged as the bunch ends distend. The beam radius decreases as the current decreases towards the head and tail of the bunch (Figure 7). The transverse temperature (angular spread) of the bunch increases as the radius goes down but not sufficiently to maintain constant emittance. 2D PIC simulations in the transverse plane have shown a slight decrease in the r.m.s. emittance $(6 \%)$ as the current ciecreases, due to rearrangement of the transverse profile as we move from the space-charge-dominated regime towards the emittance-dominated regime. There is a noticeable change in the measured transverse profile of the bunch towards a more uniform cross section in the distended bunch ends. However $2 \frac{1}{2} \mathrm{D}$ PIC simulations of the distension of the bunch ends have not shown r.m.s. emittance reduction at the level of the measurement shown in Figure 8.

\subsection{Interaction between beams}

We have checked for mutual repulsion between the beams ${ }^{10}$ and found no effect, as expected, except for a $3 \mathrm{mrad}$ deflection in the ion source which was compensated by the electrostatic design of the diode ${ }^{3}$.

\section{DESIGN OF THE LONGITUDINAL DYNAMICS}

It seems worthwhile, at this point, to explain the constraints which apply to the longitudinal motion of particles within the bunch of an ion induction linac used to drive inertial fusion.

The bunch as a whole is accelerated through the linac. In a frame moving with the longitudinal centroid of the bunch we can ignore this motion. Ignoring space charge too, for the moment, one might like to ensure 'phase-stability' by the conventional method of imposing a linear external longitudinal force directed towards the middle of the bunch, inducing phase oscillations within the bunch. This is not possible for a fusion driver such as is shown in Table 1 , because of the small momentum spread allowed by the final lens ( $\Delta p / p=10^{-4}$ at the end of the linac), for focusing onto the pellet. The bunch is so long that a particle which performs a phase-oscillation from one end to the other in the transit-time of a driver linac would exceed the allowable 
momentum spread. The burch mi $t$ have a momentum spreac so small that even in the absence of phase stability individual particles have no time to travel longitudinally out of the banch.

The length of the bunch is reduced during transit through the linac by setting up a small ordered momentum tilt which causes the bunch ends to move closer together. This iilt is to be removed before the final focus. In the bunch frame this is the only longitudinal motion deliberately imposed on the interior of the bunch.

As far as space charge is concerned, we might hope for a uniform ellipsoidal bunch which, in free space, would have linear transverse and longirudinal space charge forces. However the aspect ratio of such an ellipsoid would be extreme $(1 \mathrm{~m}$ long and $2 \mathrm{~cm}$ wide at the begining of MBE-4) and when enclosed in the conducting accelerator structure the lorgitudinal space charge force would be greatly modified. For slow variations of the line charge density $(\lambda)$ through the length of the bunch this force is then proportional to $\partial \lambda / \partial z$. It must be explicitly compensated by the external fields. During this compensation some particles may acquire a momentum error too large for the final focus.

Finally we may choose to appiy longitudinal fields to reflect back any particles which do escape from the ends of the bunch. These particles will re-enter the bunch with a momentum error which may be too large for the final focus.

\subsection{Computing the longitudinal dynamics}

In order to design the accelerating pulses and tune them in the accelerator it is necessary to compute the longitudinal dynamics. This has been accomplished by means of a simple one-dimensional computer simulation 11 'SLID' which can be run quickly on a small computer. A small number of macro-particles, e.g. 75 , are assembled with equal spacing to form a bunch. Each particle has a charge and a kinetic energy to correctly reproduce the measured bunch cuirent and kinetic energy waveforms at gap 0 . The longitudinal equations of motion are sitegrated through the linac. approximation:

The longitudinal space-charge field is calculated in the long wavelength

$$
E=-(g \partial \lambda \partial z) / \gamma^{2}
$$

$\lambda$ is the line charge density, $\gamma$ is the Lorentz factor and $g$ is a parameter including the shielding effects of the metal electrodes that surround each beam.

The longitudinal external fields are represented by instantaneous increments of the kinetic energy of each particle as it crosses an accelerating gap. These increments are given by the measured value of the accelerating voltage at the gap when the particle crosses.

The finit: width of the gap and the corresponding transit time are taken into account by numerical smoothing of the measured accelerating voltage pulses. The acceleration gap transit time at $200 \mathrm{keV}$ is $\mathbf{3 0 0} \mathrm{ns}$. Fourier components at frequencies higher than the inverse transit time of a particle of average kinetic energy are removed. 
As the bunch passes each gap a picture is built of the current and kinetic energy waveforms as functions of time, just as would be seen by a diegnostic instrument at that location.

The calcuiation is valid for a bunch in which the macro-particles are fairly evenly spaced. As two particles approach one another due to differing velocities the results become unreliable. Thus our SLID compitations are good for bunches of zero longitudinal temperature in which the particles do not overtake. Overtaking at the bunch ends occurs towards the end of the accelerator as a result of the intermittent application vit the longitudinal forces. The simple longitudinal computations described here are of little use afterwards as the kinetic energy is no longer a single-valued function of position in the bunch.

\section{1.i Determination of the g-factor}

The factor $g$ in Equaicion 1 can be determined empirically by comparing the computed current and kinetic energy waveforms for a drifting bunch with those measured. Figure 9 shows a comparison of the measured and computed current waveforn fo: a beam drifting to gap 20 . The computations are shown for three different values of $\mathrm{g}$. A single accelerating pulser firing at gap 6 has been applied to the later half of the bunch giving a velocity perturbation which evolves as a space-charge wave. By computing this evolution we can verify that the long wavelength approximation with an empirically determined $g$ fai-tor can successfully reproduce the evolution of both the mid bunch perturbation (where the line charge density and the beam radius change by a moderate amount) and the behavior of the end of the bunch (where the line charge density and the beam radius are reduced to zero). We find a value for $\mathrm{g}$ :

$$
4 \pi e_{0} g=2.2 \pm 0.2
$$

\subsection{Ideal accelerating pulses and current self-replication}

As well as tracking the longitudinal behavior of the bunch through the linac for a given set of accelerating pulses, the computations are used to initially design the schedule of acceleration and current amplification. This entails the specification of the shape, timing and amplitude of the accelerating voltage waveforms at each accelerating gap.

The bunch begins with a fairly uniform current as it traverses gap 0 . We would like the current to remain uniform while it increases during acceleration. This is called "current self-replication".11 There is a particular distribution of velocity along the bunch which, in the absence of forces acting subsequently on the particles, will preserve the shape of the current waveform, while increasing its amplitude and decreasing its duration. Expressed as a function of time and position, this velocity distribution has the form:

$$
v=(\xi-z) /(\eta-t)
$$




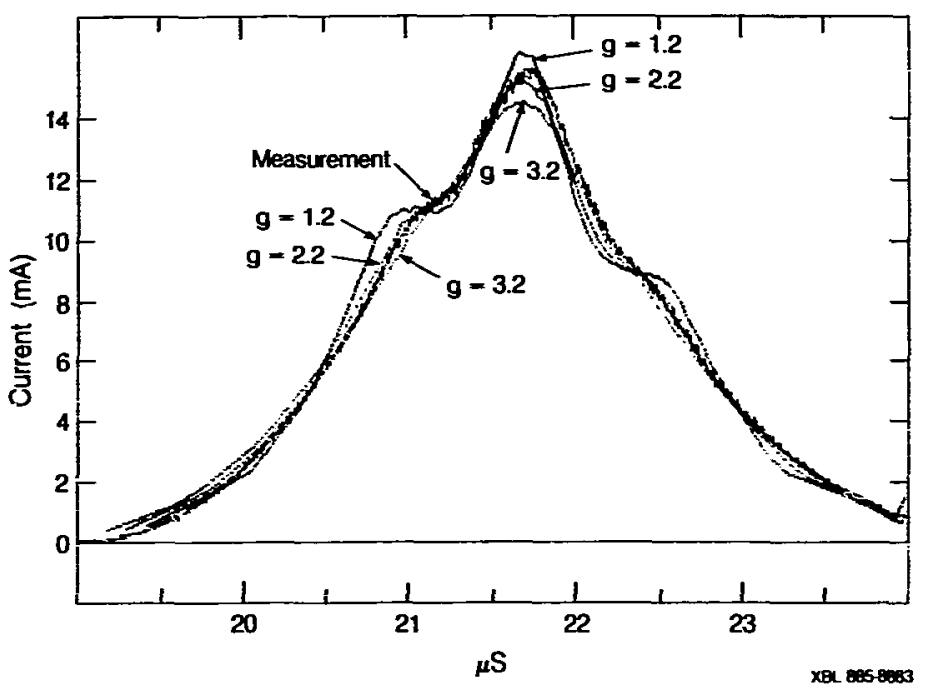

Figure 9. Current waveforms of a drifting beam, measured at gap 20, with a velocity perturbation introduced into the middle of the bunch. The longitudinal space charge forces have caused the perturbation to evolve and the bunch ends to erode. The solid lines are computations using the one dimensional simulation SLID sith $\mathbf{g}=$ 1.2,2.2 and 3.2. The computations begin from the initial conditions of Figure 2.

The two free parameters $\xi$ and $\eta$ are the coordinates of the longitudinal (virtual) focus downstream and determine the vigor with which the current and velocity increase.

We define the "ideal" accelerating voltage waveform to be the voltage required at a particular accelerating gap to accelerate and to recover this functional form for the velocity from the actual velocity distribution. The "ideal" waveform thus includes corrections for previous acceleration errors and for the uncorrected space charge effects upstream. This "ideal" waveform is not really ideal. It will try to reproduce the present shape of the current waveform, uniform or not. It includes no correction for previous acceleration errors which have been converted from kinetic energy errors into potential energy errors by the action of the space-charge forces and show up as current fluctuations, nor does the ideal waveform anticipate the effects of space-charge yet to come. This last deficiency is particularly relevant for computing the intermittent longitudinal focusing forces, where subsequent space-charge effects should be anticipated.

At each accelerating gap one invariably fails to realize the "ideal" waveform, so that the "ideal" waveforms on successive gaps become more and more intricate functions of time. In this way the "ideal" waveforms are a computational tally of the accumulated acceleration errors. 
An initial set of "ideal" accelerating waveforms was the basis for the engireering design of the induction acceleration units, descrited in Section 5. After these were installed on the accelerator an empirical tuning procedure was necessary. This will be discussed in Section 6.

\subsection{Longitudinal space charge waves}

The long wavelength approximation for the longitudinal space-charge electric field, together with the equation of continuity, give rise to a simple wave equation:

$$
\partial^{2} \lambda_{1} / \partial z^{2}=\left[\mathrm{m} /\left(\lambda_{0} g \mathrm{q}\right)\right] \partial^{2} \lambda_{1} / \partial t^{2}
$$

where $m$ and $q$ are the ion mass and charge, and $\lambda_{1}$ is 1 small perturbation to the line charge density $\lambda_{0}$.

There is no dispersion and the wave velocity in the frame moving with the bunch is:

$$
c=\left[\left(\lambda_{0} g \mathrm{q}\right) / \mathrm{m}\right]^{1 / 2}
$$

\subsubsection{Waves in MBE-4}

The bunch at the beginning of MBE-4 has $\lambda_{0}=1.9 .10^{-8} \mathrm{Cm}^{-1}$ giving : wave velocity in the bunch frame:

$$
\mathrm{c}=1.7 \mathrm{~cm} / \mu \mathrm{s} \text {. }
$$

A typical acceleration error of $1 \mathrm{kV}$ at the beginning of MBE-4 where the kinetic energy is $200 \mathrm{keV}$ gives a velocity perturbation in the bunch frame of:

$$
v_{1}^{\text {bunch }}=\left(v_{0}^{l a b} / 2\right)\left(T_{1}^{l a b} / T_{0}^{l a b}\right)=0.14 \mathrm{~cm} / \mu \mathrm{s}
$$

where $T$ is kinetic energy, $v$ is velocity, the subscript denotes the perturbation or the unpertubed quantity and the superscript denotes the frame of reference.

An acceleration error of this size becomes less significant as the beam energy increases.

Because the perturbation velocity in the bunch frame is significantly smaller than the wave velocity, acceleration frors propagate through the bunch as waves. However, at the extreme ends of the bunch where the charge density and the wave speed become small the wave picture breaks down.

The amplitude of the charge fluctuation is related to the amplitude of the velocity fluctuation in the bunch frame by the expression:

$$
\lambda_{1} \lambda_{0}=v_{1}^{\text {bunch }} / c
$$


so that a sinusoidal kinetic energy error with an ampiitude of $1 \mathrm{kV}$ will consist of two equal and oppositely moving waves which, for constant $\lambda_{0}$, will oscillate into a current fluctuation of $\pm 8 \%$ with a frequency which depends on the wavelength of the error. As $\lambda_{0}$ increases, the amplitude of the current fluctuation decreases.

We consider a typical MBE-4 accelerating error at gap 1 with a frequency of $1 \mathrm{MHz}$, giving a wavelength in the bunch frame of $0.54 \mathrm{~m}$. The resulting waves will oscillate one quarter period while the line charge density changes only slowly. The current fluctuation will be at a maximum after about $8 \mu \mathrm{s}$, when the bunch is $1 / 3$ of the way through the linac.

\subsubsection{Waves at the beginning of a driver}

We consider the effect of the same $1 \mathrm{kV}$ scceleration error, with the same frequency of $1 \mathrm{MHz}$, at the beginning or a driver, where the beam kinetic energy is $10 \mathrm{MeV}$ (Table 1). The resulting waves will now oscillate into current fiuctuations of approximately $\pm 0.2 \%$ about $25 \mathrm{~m}$ down the linac. It is clear that current fluctuations are mere easily apparent in MBE-4. Errors are more damaging when the beam kinetic energy is low, and the resulting current fluctuations are larger when the line charge density is reduced.

\section{PUISER DESIGN}

MBE-4 is a non-relativistic induction linac. It is unusual, in that the contol of the longitudinal dynamics within the bunch must be explicitly provided by the designer. The main purpose of the experiment is to explore the practical difficuities of achieving this control and of achieving the required precision in shaping the accelerating pulses. We have built two types of pulsers. At the beginning of the linac several pulsers, individually shaped and switched by thyratrons, are summed at each gap. Towards the end of the linac, larger single palsers are used, switched by spark gaps.

\subsection{Design waveforms and thyratron pulsers}

Longitudinal simulation computations have been performed to determine an accelerating voltage waveform for each accelerating gap so that the initially uniform cirrent waveform remains fairly uniform during acceleration and current amplification. 12 Figure 10 shows these waveforms for each accelerating gap. Since computations such as these depend on the details of the initial conditions, and since pulsers may have to make up tor prior errors in the acceleration schedule, the waveforms of Figure $\mathbf{1 0}$ are a first estimate of what will finally be needed.

In the first half of the linac, pulsers switched by thyratrons are employed. We do not attempt to achieve the shaped voltage on the gap with a single thyratron pulser, instead two or three pulsers are summed, giving some flexibility of shaping, since each has variable timing and amplitude. We work with two generic single-pulser voltage waveforms, each represented by simple parameterized functions. These parameterized waveforms have the following forms: 


$$
\begin{array}{ll}
V=V_{0}\left[1-\left\{\left(t-\tau_{1}\right) / \tau_{1}\right]^{2}\right] & t<\tau_{1} \\
V=V_{0} \exp \left\{\left(t_{1}-t\right) / t_{2}\right\} & t>\tau_{1}
\end{array}
$$

and

$$
\mathrm{V}=\mathrm{V}_{0}[1-\cos \pi(\mathrm{t} / \tau)]
$$

The ideal waveforms of Figure 10 are fitted by a sum of two or three of liese parameterized curves.

When the basic accelerating waveform is parameterized at each gap, simulation runs using these parametrizations are used to design the special shaping, and extra pulsers, required at various gaps to achieve longitudinal focusing.

\section{MBE-4 Ideal Waveforms}

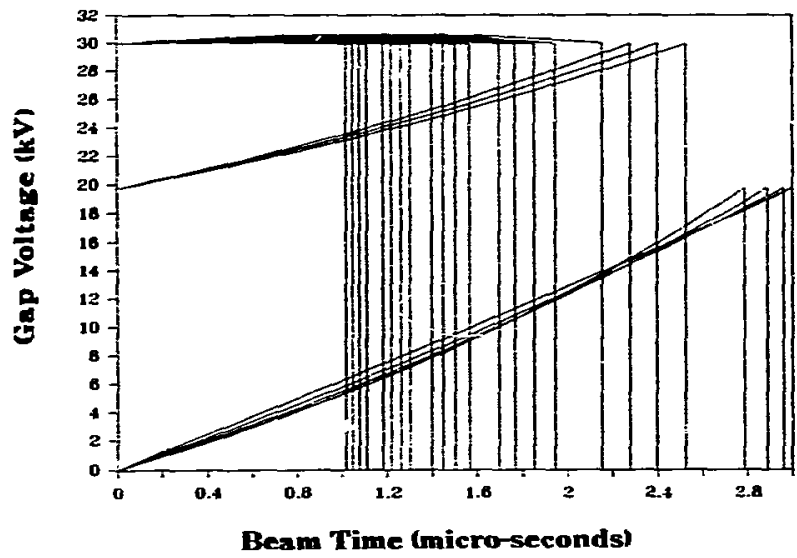

12 055-2342

Figure 10. Design accelerating voltage waveforms for each gap. These shapes are calculated without space-charge.

\subsection{Core material}

In order to keep down costs, the core material pras obtained inexpensively from various sources. Table 3 shows the arrangement of cores on each gap. 
Core arrangement, showing number of cores of each type at each accelerating gap

\begin{tabular}{|c|c|c|c|c|c|c|}
\hline \begin{tabular}{|ll}
$\begin{array}{ll}\text { core } \\
\text { type }\end{array}$ & \\
single & 5 \\
Ni-Fe & \\
\end{tabular} & $\begin{array}{r}1234 \\
3333\end{array}$ & 6789 & 11121314 & 16171819 & 21222324 & 26272829 \\
\hline $\begin{array}{ll}\text { miple } \\
\text { Ni-Fe }\end{array}$ & 3333 & $2 \cdot 21$ & 1 & 1 & 1 & 1 \\
\hline $\begin{array}{l}\text { single } \\
\text { Si-speel }\end{array}$ & & 2224 & 2 & $2 \quad 2$ & 2 & 2 \\
\hline $\begin{array}{l}\text { double } 1.4^{-} \\
\text {Si-steel }\end{array}$ & & 1 & 1 & & & \\
\hline $\begin{array}{l}\text { singke } \\
\text { Sij-skeel }\end{array}$ & & & $\begin{array}{lll}2 & 2 & 2\end{array}$ & 221 & 221 & 221 \\
\hline
\end{tabular}

\subsection{Circuit models}

Having specified the core that each thyratron pulser is to drive, and the approximate voltage waveform to be achieved, a circuit model is set up. The core is modelled as a resistor and an inductor in parallel, with values depending on voltage and empirically determined for each core-package. Pulser circuits are designed and analyzed using the circuit solving program SPICE 13 . Figure 11 shows the modeling of the two generic pulser types which were actually built. The capacitors are switchad by a hydrogen thyratron (English Electric:CX1622 or ITT:F227) and can be charged up to about 25kV. Figure 11 shows an example of the waveforms achieved. Figure 12 shows several pulsers summed on gap 11 to fit the design accelerating waveform.

\subsection{Spark gap pulsers}

From Figure 10 one can see that the last sections of MBE-4 require fiat accelerating pulses. Funhermore, because the kinetic energy has by then been significantly increased, the effect of acceleration errors is greatly reduced. For these reasons, and in order to develop broader experience with induction acceleration units, a different strategy was adopted for the last half of the accelerator. Three large $60-\mathrm{kV}$ pulsers, switched by spark gaps, were installed at gaps 17 and 18, 22 and 23, 27 and 28. Each of these large pulsers drives two core packages and the voltage waveform appears across two adjacent gaps simultancously and lasts long enough for the bunch to completely traverse the pair of gaps. This represents a rather crude attempt at matching the design waveforms, hence, smaller pulsers, switched by thyratrons, are installed ors 

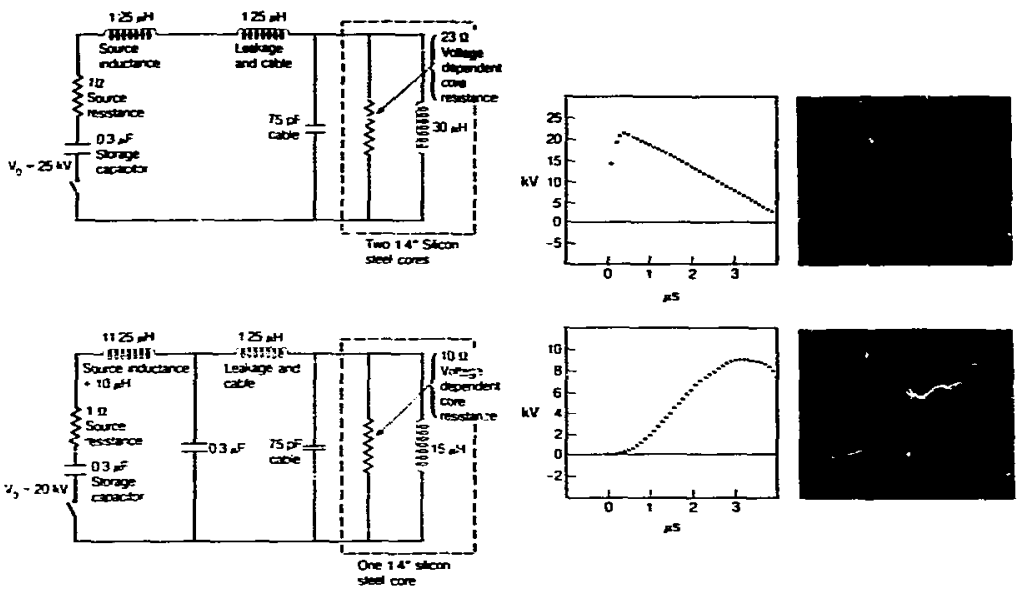

Figure 11. Modeling the two generic thyratron pulser circuits. On the left are the equivalent cincuits to be solved. Values of the components have been determined empirically. The equivalent resistance of the core depends on the charge voltage. The solutions are shown in the middle and to the right are the measured voltage waveforms.

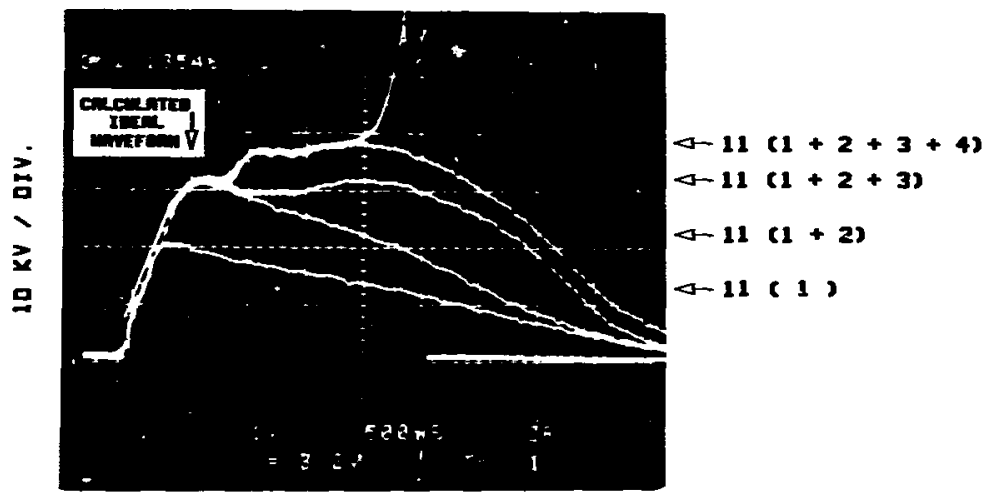

0.5 IS / 0xy.

Figure 12. An example of actual accelerating voltage waveforms summed at gap 11 to fit a design waveform. The design waveform was calculated taking into account space-charge effects and the errors on previous accelerating gaps. 
the remaining gaps $16,19,21,24,26,29$ with which to make corrections. Figure 13 shows the circuit for these spark gap pulsers, and an oscillogram of the voltage across the accelerating gap.
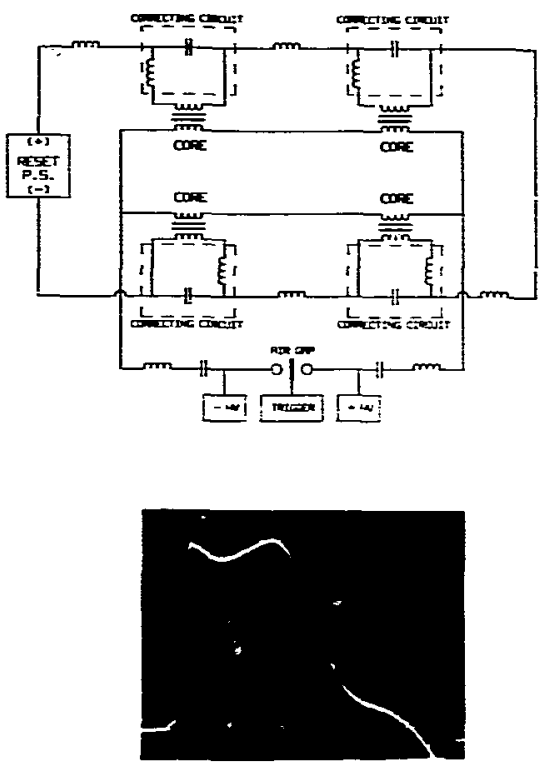

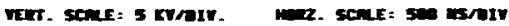

Figure 13. The circuit of the spark gap pulsers and the voltage produced at the accelerating gap. No modelling was done in this case.

\section{ACCELERATION AND CURRENT AMPLIFICATION}

\subsection{The "vigorous" acceleration schedule}

Here we describe the implementation of a vigorous acceleration schedule in which all pulsers are used close to their maximum voltages. The pulsers are designed and built based on the computed ideal waveforms. There remains some flexibility to retune the acceleration schedule to minimize current fluctuations by varying the timing 
and amplitude of the pulsers. We proceed as follows. The pulsers are tuned to be close to the schedule of voltage waveforms prescribed by the simulations. Because of the need for any given pulser to make up for the deficiencies of its neighbors and because of the way "current-self-replication" can exacerbate current fluctuations, empirical tuning is necessary, i.e., changing the accelerating voltages to minimize current fluctuations wherever they can be measured throughout the linac. A simulation run using the actual voltage waveforms on each gap acts as a guide for this tuning procedure. The calculations show precisely at what time the bunch arrives at each accelerating gap and indicate the location of voltage fluctuations corresponding to current fluctuations downstream. Figure 14 shows selected plots from such a calculation, and the correspording current waveforms measured at gaps 5, 10 and 15. After gap 20, particle-overtaking occurs and our simple simulations are no longer useful.

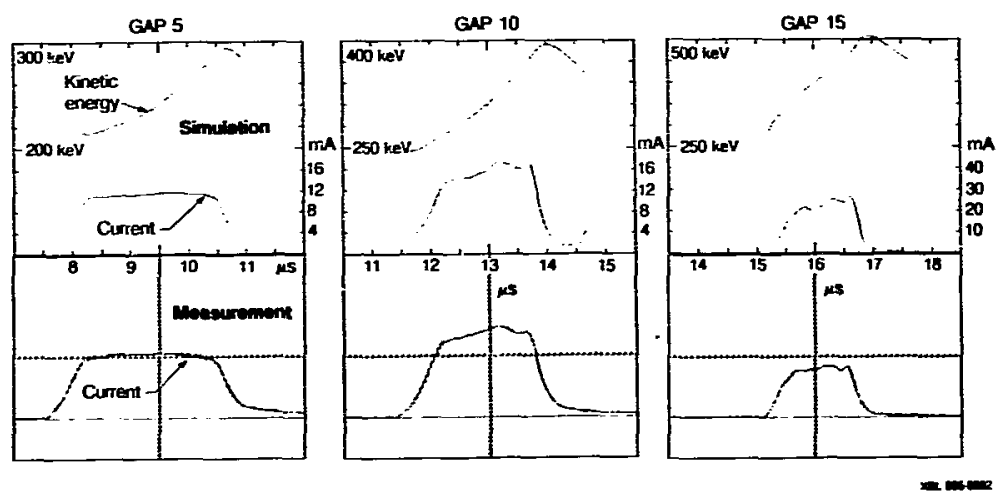

Figure 14. Simulation results using the measured accelerating waveforms to track the current and kinetic energy through the iinac. The simulations are valid only up to gap 20 where particle overtaking occurs.

Figure 15 shows the measured beam current waveforms at cach diagnostic gap when tuning is complete. No current fluctuations are seen until the wave caused by the first acceleration error has had time to oscillate one quarter wave period (see section 4.3.1). In this schedule the duration of the bunch decreases from $2.5 \mu \mathrm{s}$ to $400 \mathrm{~ns}$ and the current is amplified by a factor of 9 . Fluctuations in the current waveform of $\pm 10 \%$ are apparent. Because the risetime of the pulsers is no faster than 200 ns the longitudinal focusing produces bunch ends with approximately this duration, so that by gap 30 the head and tail account for two-thirds of the bunch length. 

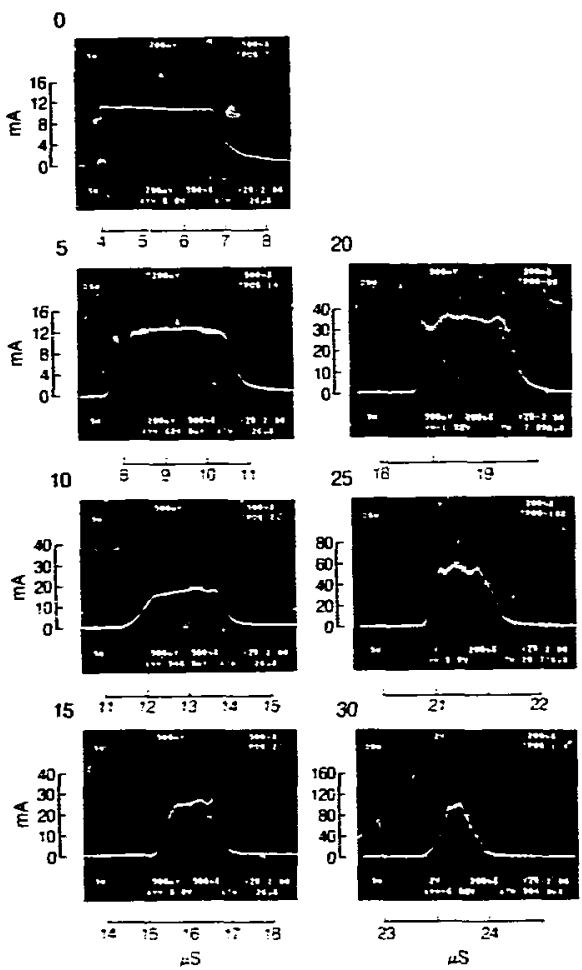

Figure 15. Measured current waveforms at each diagnostic gap under the vigorous schedule of acceleration. Several shots are superimposed.

In this schedule there is limited flexibility in the control of the bunch ends. It is particularly interesting to see in figure 15 how the ends of the bunch behave. The first longitudinal focus impulse is at gap 4. It is an extra pulser which acts to speed up the tail of the bunch. The results are seen at gap 15, the tail is steepened and a small spike appears where the focus pulser has affected the interior of the bunch. Subsequently the tail slows and spreads under the longitudinal space charge force. The first focus impulses on the head of the bunch are at gaps six and seven. Here we modify the main pulsers to refrain from accelerating the head of the bunch, causing the head to slow, and steepen at gap 20. Again a small spike appears where the focus impulse has intruded into the body of the bunch. Subsequenty the head speeds up and spreads under the longitudinal space charge force. 
Figure 16 shows the current waveforms for all four beams at gap 30 . The longitudinal dynamics is the same for all of the beams.
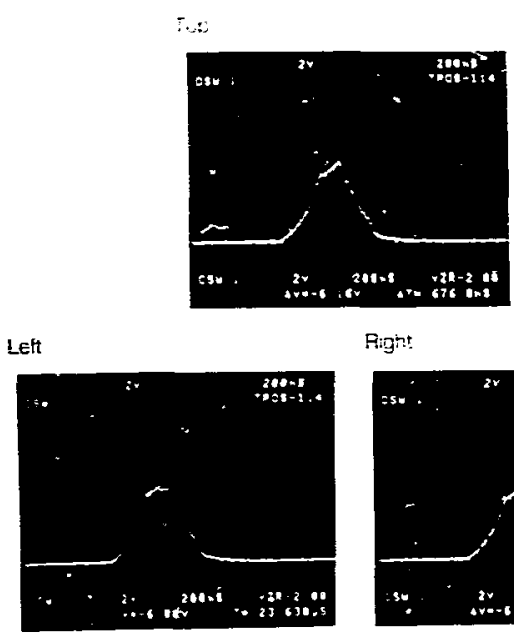

Pigint

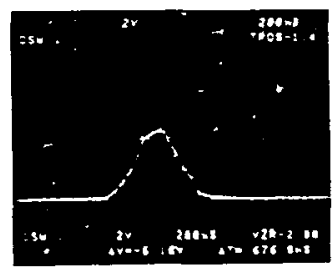

Sat:om

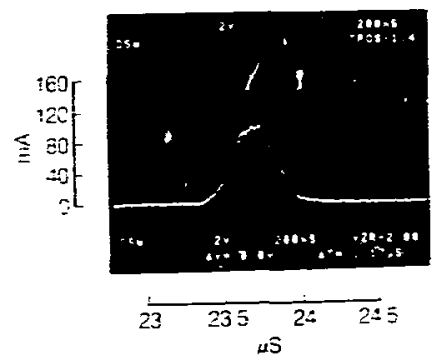

Figure 16. Current waveforms of all four beams at gap 30 under the vigorous schedule of acceleration. Several shots are superimposed.

\subsubsection{Final longitudinal emittance}

We have measured the longitudiral emittance at gap $\mathbf{3 0}$ after acceleration. The beam is collimated by a narrow horizontal slit (.003") and deflected onto a narrow sense 
wire where the incident current, amplified by secondary electron emission, is observed. The system is sensitive to ions with kinetic energy within $0.3 \%$ of a central value which depends on the magnitude of the deflecting electric field. The detection system has a time resolution of about \pm 10 ns. We observe several shots at a fixed deflecting voltage to include the effect of shot-to-shot variations in our measured emittance. The deflecting voltage is then changed to build up a picturs of the longitudinal emittance.

Figure 17 shows the results. The shot-to-shot variations contribute to the width of the distribution, although in the narrowest regions these variations are unresolved. The systematic acceleration errors are a more important source of longitudinal emittance, giving kinks in the plot which contribute to the area of an ellipse drawn around the distribution.

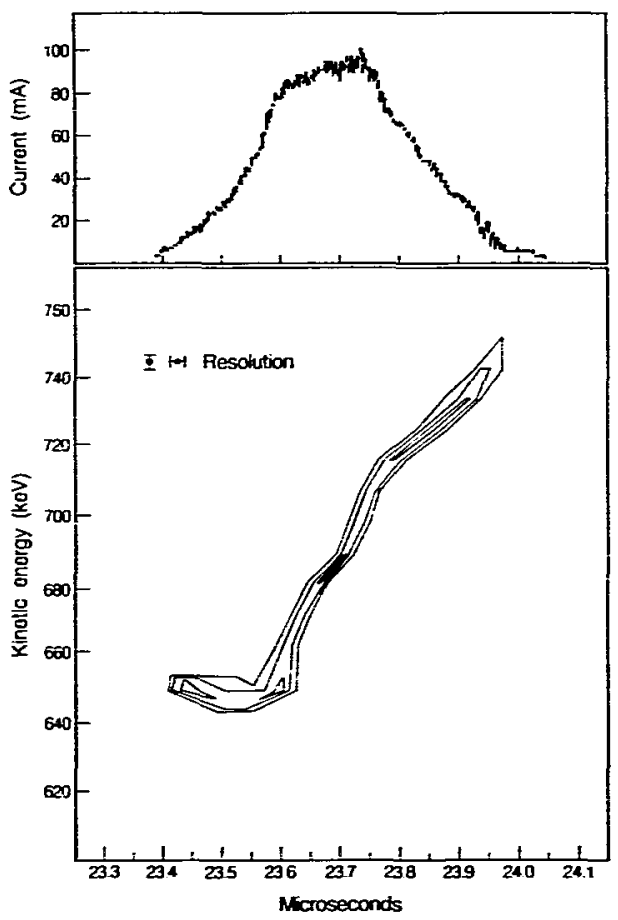

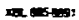

Figure 17. Measured longitudinal phase space distribution for one of the four beams aseilerated vigorously to gap 30 . The contours are logarithmic and include the effect of shot-to-shot variations. If one encloses the distribution in an ellipse the area is approximately $4 \pi \cdot 10-3 \mathrm{eV}$ s. 
Disregarding the large systematic error at the head of the bunch, which could have been corrected if more pulsers had been available, we estimate the area of such an ellipse to be:

$$
\pi \varepsilon_{\text {longiudina }}=4 \cdot 0 \cdot 10^{-3} \pi \mathrm{eV}-\mathrm{s}
$$

\subsection{The "gentle" acceleration schedule}

In the interest of more flexibility to correct acceleration errors and to control the bunch ends in MBE-4, we have developed a gentle acceleration schedule in which several of the acceleration gaps are devoted exclusively to making small acceleration corrections.

We envision that in a large fusion driver one would want to make acceleration corrections at intervals spaced down the accelerator so that preceding acceleration errors could be corrected before they develop into current fluctuations. (These intervals may be about ten meters long at the beginning of a driver, see section 4.3.2). This would allow one to build pulsers which were less flexible, in the main, provided the resulting accelerating voltage errors could be precisely removed at the correction station. We imagine the correction pulsers, of small anplinude, would be rather flexible and could be more expensive and/or less efficient if necessary. These interspersed correctors would also be the natural place to apply voltages to control the bunch ends.

In the gentle schedule on MBE-4 gaps 9, 11, 16, 21 and 26 are to be correction locations, given that current fluctuations will emerge a few meters downstream from the original error (4.3.1). We have installed small-amplitude pulsers which can be turned off and on, reverseci in polarity and moved around in time and amplitude. Figure 18 shows an example of one of these correction voltage waveforms.

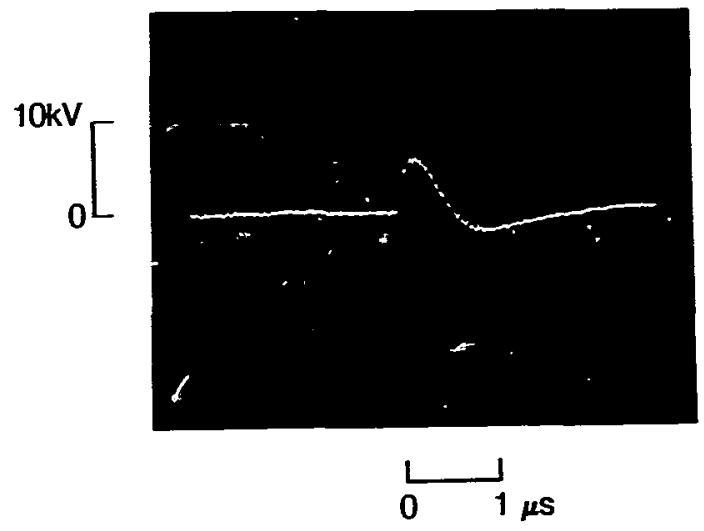

Figure 18. An example of the individual correcting voltage pulses, three or four of which are used at several accelerating gups in the gentle accelerating schedule. 
The price to be paid in giving up core material for correctors is a somewhat reduced average accelerating gradient and a lower final kinetic energy.

We also implement a lower factor of current amplitication. The beamlet current increases from $10 \mathrm{~mA}$ to $35 \mathrm{~mA}$ in this schedule.

The tuning procedure is similar to that for the vigorous schedule. The simulation code SL.DD is used to design the schedule using "ideal" waveforms and zero voltage at the corrction gaps. Pulsers are tuned on all garis except the correction locations and SLID can comfute the longitudinal dynamics orice more, this time using the actual accelerating voltage waveforms at the accelerating gaps and 'ideal' waveforms at the correcting gaps. This gives an idea of the corrections required. The correction pulsers are finaily tuned by looking at the current waveforms throughout the linac itself.

Figure 19 shows a selection of the accelerating voltage waveforms used for the gentle schedule. The bar under each voltage waveform shows the timing of the bunch at that gap. At gap 3 we are partially correcting the kinetic energy profile for the diode transient effects (see Figure 2). At gaps 6 and 8 we apply a voltage which is ramped, to establish the velocity tilt from head to tail for current amplification. Gap 9 (not shown) is the first correction gap, where the longitudinal space charge effects at the
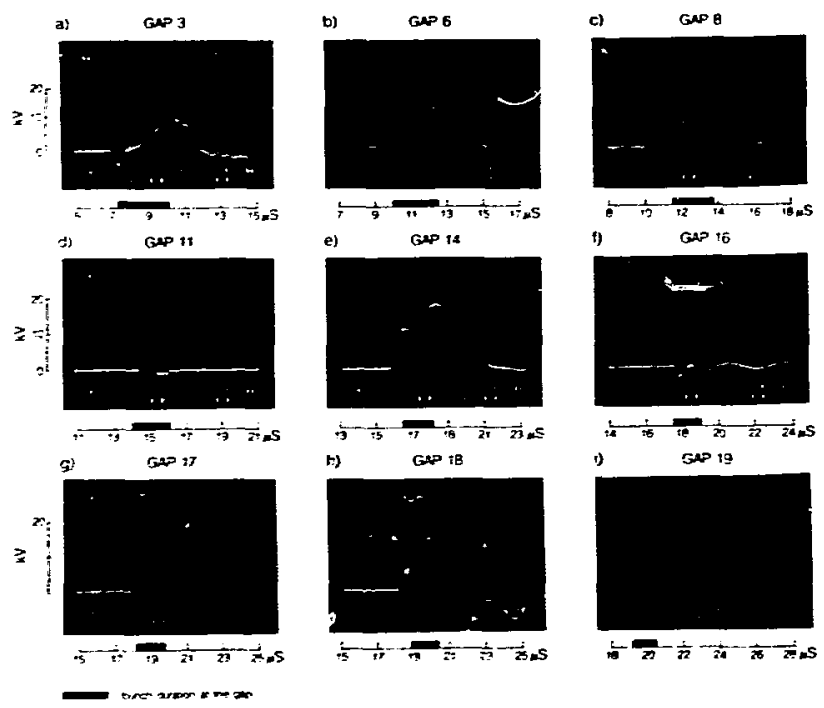

Figure 19. Composite accelerating voltage waveforms at several gaps, used for the gentle accelerating schedule. The solid bar vider each picture shows the timing of the bunch at that gap. 
bunch ends are empirically compensated. Gaps 11 and 16 are the next correction gaps, several small correction pulsers are shown, tuned empinically to minimize current fluctuations. Gap 14 is ramped up to compensate for gaps 12, 13 (not shown) which were ramped down due to core configuration constraints. Gaps 17 and 18 are driven by the same large spark-gap pulser and their voltage waveforms appear simultaneously. However the bunch arrives first at gap 17 then at gap 18, as shown, and the tail of the bunch sees a rapidly falling voltage at gap 18. Gap 19 is tuned to make up for this deficiency at gap 18; and so on through the linac to gap 30.

Figure 20 shows the current waveforms measured at each diagnostic gap under this schedule.
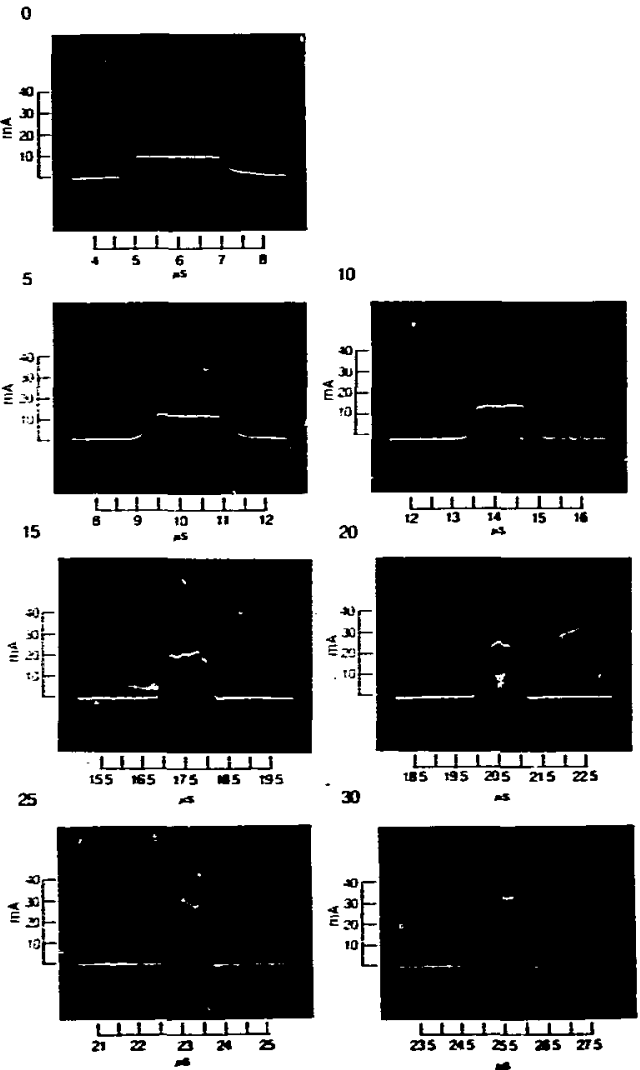

Figure 20. Measured current waveforms at each diagnostic gap under the gentle schedule of acceleration. Several shots are superimposed. 


\subsubsection{Final longitudinal emittance}

The longitudinal emittance is measured at the end of the linac and is shown in figure 21. The contours are logarithmic in intensity. The measurement is made over abont 100 shots and includes the shot-to-shot variations in kinetic energy and arrival time. These shot-to-shot variations arise from small variations of the voltage pulses from the accelerating modules and give rise to the finite width of the distribution in figure 21. Some systematic perturbations are observable at the bunch ends, but much less than in the vigorous schedule. At the ends the kinetic energy is not a single-valued function of the arrival time, even in a single shot, due to the effects of the correcting pulsers which are used for control against the longitudinal space charge force. The area of an ellipse surrounding this distribution is again set by the systematic acceleration errors and is estimated to be:

$$
\pi \varepsilon_{\text {longitudinal }}=3.0-10^{-3} \pi \mathrm{eV}-\mathrm{s}
$$

which is $75 \%$ of the value previously obtained.

We now address the relationship between the longitudiral emittance achieved in MBE-4 and that required in a fusion driver.

The uncorrelated acceleration errors acting, at each gap, on a particular slice of the bunch length, contribute to the final momentum spread like a one dimensional random walk with a step size decreasing down the linac. The contribution is largest from the beginning of the accelerator because of subsequent acceleration and subsequent bunch shortening.

In order to derive a simple expression for the fina' momentum spread ${ }^{15}$ we assume that the voltage $(\Delta \mathrm{V})$ applied to the beam is the same at each gap, with uncorrelated errors having the same root mean square magnitude $\left(\lambda\left\langle\eta^{2}\right\rangle \Delta V\right)$ where $\eta$ is the fractional voltage error. We parametrize the bunch length through the linac as

$$
1 \sim V^{-1 / 4}
$$

Then the final r.ms. momentum spread is given by:

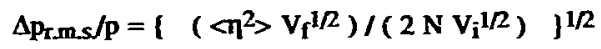

where 1 is the number of accelerating gaps, $V_{f}$ is the beam kinetic energy at the end of the linac and $V_{i}$ is the kinetic energy at injection.

In $M B E-4, V_{i}=200 \mathrm{keV}, V_{\mathrm{f}}=620 \mathrm{keV}, \mathrm{N}=21$ and the maximum accelerating voltage error is about $2.5 \%$, giving $\langle\eta 2\rangle \approx 2 \cdot 10^{-4}$ for a uniform distribution of random error amplitude with zero mean. Using these parameters in the formula above and the bunch duration from Figure 21 gives

$$
\pi \varepsilon_{\text {longindinal }}=3.6-10^{-3} \pi \mathrm{eV}-\mathrm{s}
$$

which is close to the measured value. 
Using the same accelerating voltage and accelerating errors in a fusion driver with $V_{i}=10 \mathrm{MeV}$ and $V_{f}=10 \mathrm{GeV}$ (Table 1) gives $N=1.5 \cdot 10^{5}$ and

$$
\Delta \mathrm{p}_{\mathrm{r} \text { m.s }} / \mathrm{p}=1.5 .10^{-4}
$$

This is close to the maximum value of 1 to 2.10-4 that would be allowed under the constraints of the final focus onto the fusion target. This analysis is consistent with an earlier estimate of the accelerating voltage errors allowable in a driver, discussed by Faltens and Keefe ${ }^{16}$, who concluded that voltage errors had to be controlled to $1 \%$. It should be noted that in this scenario there is no phase space available for longindinal emittance growth through the interaction of high current beams with the accelerator impedance.

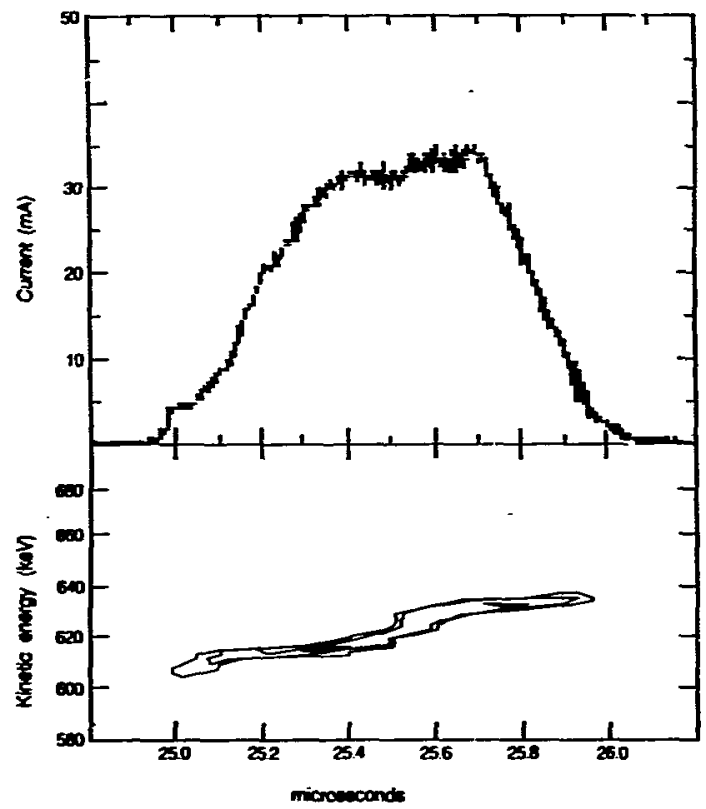

Figure 21. Measured kngitudinal phase space distribution for one of the four bcams accelerated gently to gap 30 . The contours are logarithmic and include the effect of shot-10-shot variations. If one encloses the distribution in an ellipse the area is approximately $3 \pi \cdot 10-3 \mathrm{eV}$ s. 


\subsubsection{Transmission}

The transmission was measured under the gentle schedule by digitizing current measurements at each diagnostic station and integrating numerically. There is a tail to the bunch at gap 0 (see Figure 3) which is not accelerated and falls behind (Figures 15, 20), until it can no longer be detected. We are concerned with the transmitted fraction of the bunch proper, the loss of the tail under acceleration is not of interest. In Figure 22 we plot the transmitted charge integrated from the head of the bunch up to that point where the tail begins, the knee in the curve of integrated charge versus time, with error bars to reflect the uncertain location of this point. We conclude that there are no other measurable losses during acceleration under the gentle schedule.
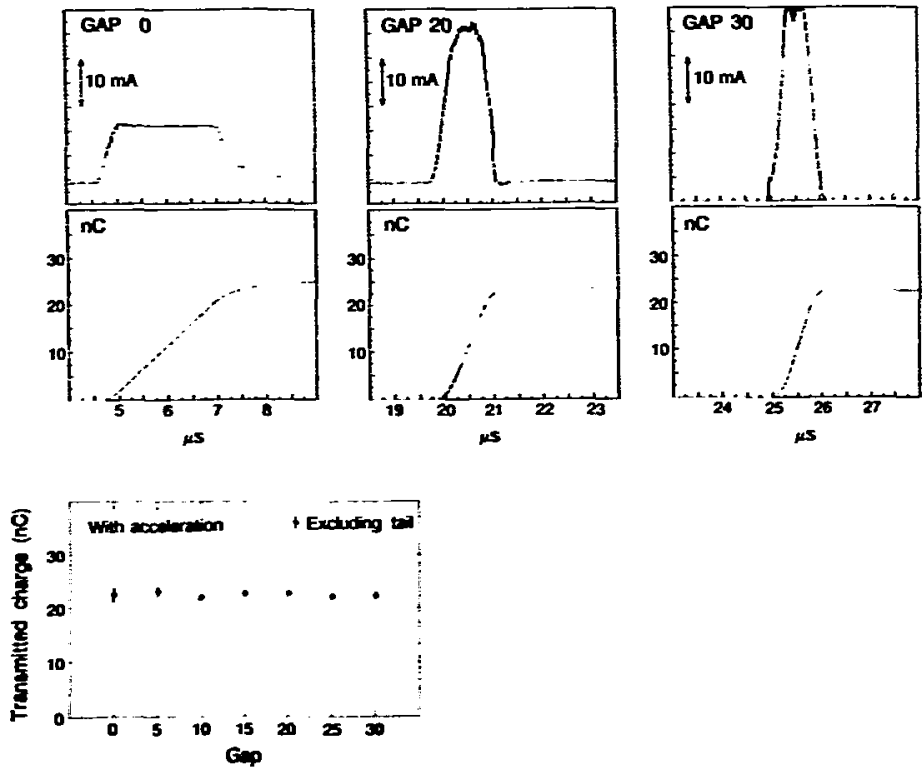

Figure 22. Transmission under the gentle acceleration schedule. The current measurements are integraied from the arrival time of the head of the bunch to the arrival time of the tail and one can see a knee in the curve of integrated charge where the tail, which is not accelerated, begins. We plot the integrated charge up to the point where the tail begins, measured at each diagnostic location. Compare these numbers with the transmitted charge for the unaccelerated beam shown in Figure 4, there are no measurable additional losses during acceleration. 


\subsubsection{Final transverse emittance}

Figure 23 shows time-resolved measurements of the un-normalized transverse emittance at the end of MBE-4, a) for a drifting beam at $200 \mathrm{keV}$ with no acceleration and b) for a beam accelerated through the gentle schedule to $620 \mathrm{keV}$. These data are for a slice of about $100 \mathrm{~ns}$ duration at the detector, midway between head and tail of the bunch. Because of the increase in velocity, the un-normalized emittance in b) should be reduced by a factor of 1.75 . These data show essentially no reduction, implying emittance growth by approximately this factor during acceleration. At this point we have not accelerated a beam at full current through MBE-4 without observing some growth of the transverse emittance. Work is continuing to identify the source of the growth
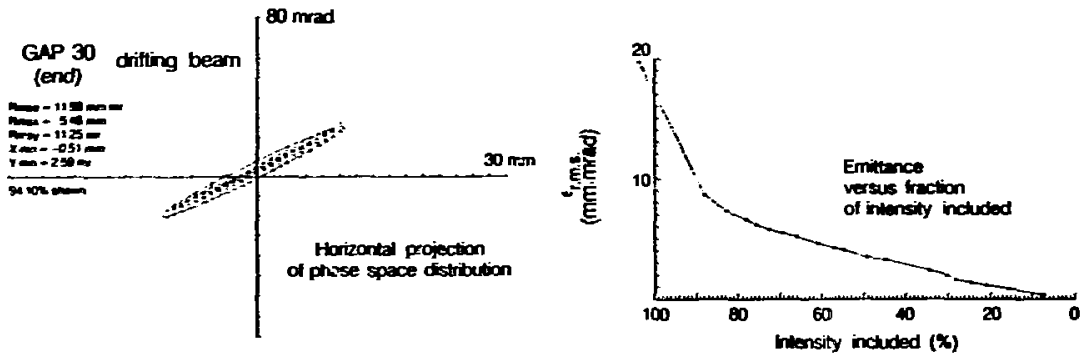

GAP 30 gentle acceleration schedule
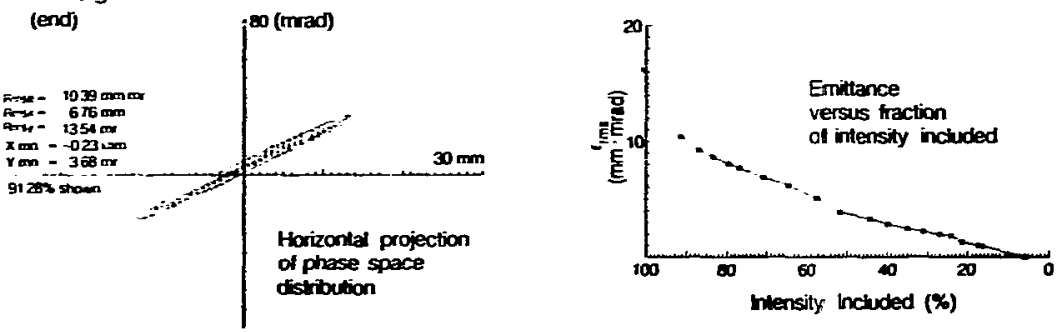

incentse

Figure 23. Transverse cmitrance at the end of the linac. The un-normalized emittance is plotted for the drifting beam and for the beam accelerated through the gentle schedule. The phase space distribution at the longitudinal center of the bunch is shown os the left. On the right the emittance is plotted versus the fraction of the intensity, above a varying phase space density threshold applied before the rm.s. computation. 


\section{RESULTS}

We have demonstrated that the principle of acceleration and current amplification of multiple ion beams in an induction linac is workable.

The short bunch employed in this experiment over-emphasizes the problems of bunch end control, in that the ends of the accelerated bunch represent two-thirds of its overall length. We have best achieved control over the longitudinal space-charge forces at the ends by ernpirical tuning of small accelerating pulsers located at several correcting stations through the linac. This was also found to be the best way to minimize current fluctuations in the body of the bunch.

Acceleration enrors we have accumulated represent an increase of the longitudinal emittance which is close to the maximum allowable. However, systematic acceleration errors, which are the same every shot, determine this experimental result. One might hope, in the future, to be able to remove such errors. They could not easily be removed here because of the limited number and the limitud frequency response of the accelerating pulsers.

We have observed growth of the transverse emittance by about a factor of two as the full current beam is accelerated through MBE-4. Work is continuing to locate the origin of this growth.

We have observed an unexplained reduction of the transverse emittance in the ends of the bunch as they are distended by the longitudinal space charge fieids.

We find that the K-V envelope equations reflect the radii of the beams quite accurately (to within about a millimeter out of about 12 millimeters), although we observe non-uniform phase space distributions in every measurement.

The one dimensional model of the longitudinal beam dynamics in which the space-charge force is proportional to $\delta \lambda / \partial z$, with an empirically determined constant of proportionality (g), has been a vseful tool for design and tuning. The implementation of this model as a one dimensional simulation was limited by the inability to handle overtaking of the macroparticles. It may be that a more sophisticated implementation of this model is all that is required for the future.

\section{CONCLUSIONS}

It would not be reasonable to conclude this preliminary report without commenting on the practical problems we have encountered while running MBE-4.

We have suffered throughout this work from quadrupole misalignments. The design called for mechanical tolerances on the four fold quadrupole structure of about 0.004 inches. We have measured positional enors at times as large as .020 inches. The structure moves during pumping and in response to temperature changes between early morning and lare afteranon. The most serious consequence of this is the limit on useful betatron tunes. In order to transport the accelerated beam clear of the quadrupole electrodes we would have liked to have been able to use tunes as high as 90 degrees but could not, because of the large amplitude coherent betatron oscillations which then arise out of the quadrupole misalignments. 
Reliability of the accelerating pulsers has been an issue. The acceleration schedules typically use about 70 pulsers. Failure of one or more of these was common.

Although the thermionic ion sources emit at their space charge limit over a period of months without attention, the thermal cycles cause motion in the diode which is compensated, as possible, by steering at the exit of the gun. This tuning is difficult and laborious, so that we often worked with only one of the four inn beams well aligned at the entrance to the linac. Improved diagnostics would have helped here. The prospect of controlling a large number of beams is sobering.

Our beam measurement techniques throughout the linac were time consuming. In a future linac with more than four beams, considerable attention should be given to developing convenient measurement techniques.

The limited frequency response of the induction modules causes concern. In MBE-4 this restricted the duration over which we could control the bunch ends. The limiting component was the accelerating cores themselves, which for reasons of cost, were not made of the ideal material. However, future machines may need significantly faster response (see Table 1) and a lumped element driving circuit may be inappropriate. We will give up what little shaping flexibility we presently have when we have to build driver circuits using transmission lines.

This brings us to the need for flexibility and tunability in the accelerating pulsers. This is difficult and expensive to achieve, but is certainly required. It is required either to match the computed waveforms, in a situation where a precise and realistic numerical model of the longindinal dynamics is applied to the tuning problem, or altematively to tune empirically to rinimize current and energy fluctuations measured in the accelerator.

We must also consider the probability that fluctuations of the beam current in loaded accelerating modules, running with high efficiency, would grow through interaction with the longitudinal impedance of the structure. ${ }^{17}$ If the growth rate is not too large, the damping of this instability could be incorporated in the tuning procedure. This could be accomplished either by including the growth in a precise model used to compute the waveforms, or, more practically, by empirical tuning to minimize current fluctuations. In either case great flexibility would be required of the pulsers.

We suggest the following procedures with the accelerating induction pulsers for a fusion driver:

i) Build low voltage pulsers, say $50 \mathrm{kV}$, for the first several hundred meters of 2 driver where acceleration errors are most important.

ii) Build 'crude' pulsers which can somehow generate the required accelerating waveforms to about $1 \%$, ignoring special requirements for error correction or for control at the bunch ends.

iii) Build flexible low amplitude correction pulsers to insert at intervals throughout the linac. The spacing of these correctors depends on the frequency of typical accelerating errors and on the space charge wave speed in the beam bunch. These pulsers should be able to act on the bunch ends and on acceleration errors before they oscillate into current fluctuations.

iv) After turning on the crude pulsers, tune the correctors. This conld be done in response to precise and realistic computations of the longitudinal dynamics which take into account everything upstream, including the longitudinal 
impedance, or, more practically, it could be done empirically, to minimize current fluctuations a suitable distance downstream.

v) These procedures will only be feasible if the longitudinal growth rates are not too large.

\section{REFERENCES}

1. A. Faltens, E. Hoyer and D. Keefe, Proc. 4th Int Top. Conf. High-Power Electron Ion Beam Res. Tech., Palaiseau, 1981, p. 751, J. Hovingh, V. Brady, A. Faltens, D. Keefe and E. Lee, Fusion Technology 13 (1988) p. 201.

2. "Alumino-Silicate Ion Sources for Accelerator Applications", A.I. Warwick, Proc. Particle Accelicator Conf., Vancouver, May 1985.

3. "A Four Beam Cesium Injector for MBE-4", A. I. Warwick, D. Vanecek and O. Fredriksson, Proc. Particle Accelerator Conf., Vancouver, May 1985.

4. "MBE-4, A Heavy Ion Multiple Beam Experiment", R.T.Avery, et. al., Proc. Particle Accelerator Conf., Vancouver, May 1985.

5. "Linear Envelope Calculations for MBE-4", A. I Warwick, Lawrence Berkeley Laboratory internal note, HIFAR-174, Oct 1987.

6. "Pulsers for the Induction Linac Experiment MBE-4", D. Gough, D. Brodzik, A. Faltens, C. Pike and J. Stoker, Proc. Particle Accelerator Conf., Washington D.C., March 1987.

7. "Bunch End Erosion", A. Faltens, E. P. Lee and S. Rosenblum, J. Appl. Phys., 6112 (1986) 5219.

8. "Transverse Emittance Measurement Techniques for MBE-4", A.I.Warwick, Lawrence Berkeley Laboratory internal note, HIFAR-185, Feb 1988.

"Accelerator Research on MBE-4, An Experimental Multi-Beam Induction Linac." H. Meuth, T. J. Fessenden, D. Keefe and A. I. Warwick, Proc. Intl. Symposiam on Heavy Ion Inertial Fusion, Darmstadt, June 1988.

9. LM.Kapchinskij and V.V.Vladimirskij, Proc. 2nd. Int. Conf. High Energy Accel., p. 274 (1958)

10. "Preliminary Results from MBE-4: a Four Beam Induction Linac for Heavy Ion fusion research", T. J. Fessenden, D. L. Judd, D. Keefe, C. Kim, L. J. Laslett, L. Smith, and A. I. Warwick, Proc. Int. Symp. on Heavy Ion Fusion, Washington DC, p. 145 (1986) 
11. "Ion Induction Linac Design and Operation Codes, INDEX/SLID, Users Manual", C. H. Kim, private communication.

"A Design Procedure for Acceleration and Bunching in an Ion Induction Linac", C. H. Kim and L. Smith, Particle Accelerators 18 (1985)i01.

12. "Synthesis of MBE-4 Accelerating Waveforms", C. H. Kim, V. O. Brady, T. J. Fessenden, D. L. Judd and L. J. Laslett, Proc. Particle Accelerator Conf., Vancouver, May 1985.

13. "Spice User's Guide", T. Quarles, A. R. Newton, D. O. Pederson and A. Sangiovanni-Vincentelli, Dept. of Elec. Eng. report, Univ. of Calif., Berkeley.

14. These resuits have been published in the literature:

H. Meuth etal., Nicl. Inst. Meth. in Phys. Rés. A278(1989) p. 153.

A. LWarwick et.al., Proc. Europ. Part. Accel. Conf., Rome, June 1988, p. 118.

A. LWarwick et al, Proc. Linear. Accel. Conf., Williamsburg, Oct.1988, p. 51.

15. "Acceleration Errors in MBE-4 and in a Driver", A. Warwick and E. P. Lee, Lawrence Berkeley Laboratory internal note HIIFAR-214, Oct. 1988

16. "Power amplification of a heavy ion beam in an ion induction linac" A. Faltens and D. Keefe, Proc. 5th Int. Conf. on High Power Particle Beams, San Francisco, 1983, p. 314 ; LLNL-CONF-830911.

17. "Resistive Instability Growth Rates", L. Smith, Lawrence Berkeley Laboratory internal note, HIFAR-217, Sept. 1988. 